\title{
1. Identitätspolitiken: Queer-feministische Debatten
}

Einer queeren Kritik an (eindeutigen) Identitätspolitiken gehen viele aktivistische wie theoretische Debatten voraus. Im Folgenden werden einige feministische, intersektionale und postkoloniale Perspektiven der 1980er- und 1990er-Jahre skizziert. Dadurch soll einerseits ein Blick auf frühe Debatten und Interventionen zu identitätspolitischen Dilemmata ermöglicht werden und andererseits auf die Perspektiven ${ }^{1}$ verwiesen werden, die mittlerweile - wenn auch zu einem unterschiedlichen Grad - Eingang in queere Politiken und Theoriebildungen gefunden haben. Hierzu wird im Folgenden entlang einzelner Debatten, wie beispielsweise des »Streits um die Differenz«, oder entlang einzelner Interventionen, wie beispielsweise Spivaks Aufsatz "Can the Subaltern Speak?«, der Blick auf feministische, postkoloniale und intersektionale Perspektiven geworfen. Im Anschluss wird entlang der theoretischen Bewegung von der Heteronormativität zur Homonormativität bis hin zum Homonationalismus der Blick auf queere Theorien und Auseinandersetzungen gelegt.

\subsection{Poststrukturalismus und Kritische Theorie: Der Streit um die Differenz}

Das Erscheinen von Judith Butlers bekanntem Werk »Gender Trouble «1990 ${ }^{2}$ hat sowohl im deutschsprachigen als auch im US-amerikanischen Raum für kontroverse Debatten gesorgt. Eine solche frühe Debatte in den USA mündete in der Aufsatzsammlung mit dem Titel »Der Streit um die Differenz«. Dort diskutieren die »bekanntesten feministischen Theoretikerinnen der USA« (Seyla Benhabib, Judith Butler, Nancy Fraser und Drucilla Cornell) - so der Klappentext des 1993 auf Deutsch veröffentlichten Buches die Rolle, die die sogenannte Postmoderne für den Feminismus spielen kann. Vorausgegangen ist diesem Streit unter anderem Judith Butlers Kritik an der Kategorie »Frau als Subjekt des Feminismus«: $:^{3}$ In »Gender Trouble« kritisiert sie, dass ein politisches Sub-

\footnotetext{
Hiermit sind insbesondere intersektionale und postkoloniale Theorien gemeint. 1991 ist die deutsche Übersetzung »Das Unbehagen der Geschlechter« erschienen. Seyla Benhabib/Judith Butler/Drucilla Cornell/Nancy Fraser: Der Streit um Differenz. Feminismus und Postmoderne in der Gegenwart, Frankfurt a.M.: Fischer Verlag 1995.
} 
jekt, das von einer bereits unterworfenen Identitäts-Kategorie - „Frau« - ausgeht, so agiert, als wäre Identität immer schon da und vorausgesetzt. Dadurch werde verschleiert, dass Identitäten erst in und durch Handlungen (wie Identitätspolitiken) hergestellt werden. ${ }^{4}$ Den Bedingungen der sprachlichen Repräsentationen, die festlegen, was ein Subjekt sein kann, damit es repräsentierbar ist, könne - so Butlers Kritik - so nicht entkommen werden. Dies sei besonders dann problematisch, wenn Subjekte entlang differentieller Herrschaftsachsen hervorgebracht werden. ${ }^{5}$ Doch auch Butler betont, dass es nicht möglich sei, gänzlich auf Repräsentationspolitiken zu verzichten. ${ }^{6}$ Es reiche aber ihr zufolge auch nicht aus, die Kategorie »Frau« »einfach mit verschiedenen Bestandteilen wie Bestimmungen der Rasse, Klasse, Alter, Ethnie und Sexualität « zu vervollständigen. ${ }^{7}$ Stattdessen solle es darum gehen, die »wesentliche Unvollständigkeit dieser Kategorien « vorauszusetzen, indem das politische Subjekt selbst zur Verhandlung gestellt wird. Butler möchte folglich das Feld des Politischen verschieben: weg von einem Feminismus, der das Subjekt Frau voraussetzt, hin zu einer Politik, in der Identität als "methodische und normative Voraussetzung "verstanden wird und in der entsprechend eine veränderte Konstruktion von Identität selbst das Ziel ist. ${ }^{8}$

\section{Der Tod des Subjekts: Das Ende feministischer Politik?}

Die stärkste Gegenposition im »Streit um die Differenz« vertritt Seyla Benhabib, die als eine Vertreter*in der Kritischen Theorie gilt. Benhabib verortet Butlers Interventionen in einer postmodernen Theorietradition und fragt, welche Rolle ein postmoderner Feminismus politisch spielen könne. ${ }^{9}$ Ausgehend von dem »Tod des Subjekts«, dem »Tod der Geschichte « und dem »Tod der Metaphysik«, den postmoderne Theorie ihr zufolge markiert, unterscheidet sie jeweils eine starke und eine schwache Version dieser drei Tode. Zumindest eine starke Version ist Benhabib zufolge unvereinbar mit feministisch-emanzipatorischen Politiken. Bezug nehmend auf die These vom »Tod des Subjekts« stellt sie in Frage, ob »das Projekt weiblicher Emanzipation ohne ein solches regulatives Prinzip der Handlungsfähigkeit, der Autonomie und der Ich-Identität überhaupt denkbar« sei. ${ }^{10}$ Auch die mit dem »Tod der Geschichte« einhergehende Unmöglichkeit, eine alternative feministische Geschichte $\mathrm{zu}$ schreiben, steht dem feministischen Projekt Benhabib zufolge entgegen. Das »Ende der Metaphysik« bedeutet in ihren Augen wiederum ein Ende der Möglichkeit einer feministischen Gesellschaftskritik. In einer postmodernen Absage an die Geschichte, die Metaphysik und das Subjekt - so Benhabib - kumuliert die Unmöglichkeit, feministische Utopien zu formulieren. So schließt sie ihren ersten Aufsatz im »Streit um die Differenz« mit dem Appell, die utopische Hoffnung nicht aufzugeben: "gerade wir - als Frauen - haben viel zu verlieren, wenn

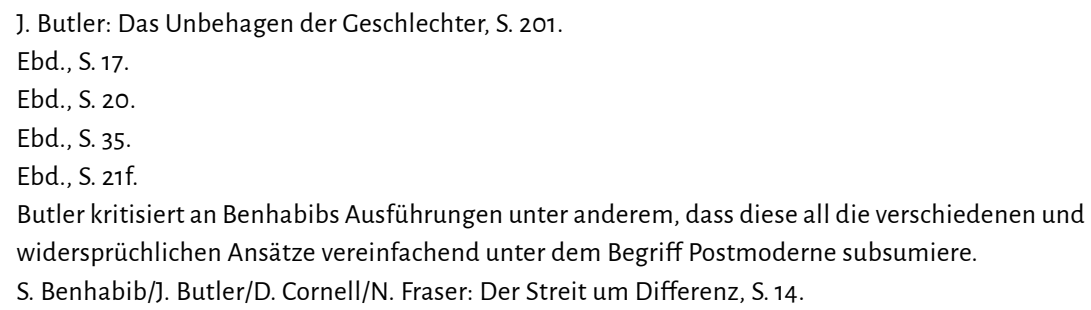


wir die utopische Hoffnung in das ganz andere aufgeben. «" Hier setzt Benhabib bewusst das feministische Subjekt »Frau« ein, um sich im Namen des Prinzips Hoffnung gegen das Ende eines um Identität angeordneten politischen Subjekts »Frau« zu wenden. Benhabib vertritt in diesem Aufsatz die Meinung, dass der Feminismus nicht ganz auf ein Subjekt, eine Geschichte und Gesellschaftskritik verzichten kann, will er seine emanzipatorische Kraft behalten. ${ }^{12}$ In späteren Auseinandersetzungen führt sie zudem die Unterscheidung zwischen einer dritten Beobachter*innenposition einerseits, die eine konstruktivistische Kritik an einem um Identität angeordneten politischen Subjekt (auch ohne die drei Tode anzunehmen) ausüben kann, und einer »Perspektive der ersten Person « andererseits, von der ausgehend Identität von Bedeutung sein muss, ein: »Mitglieder und Theoretikerinnen von Bewegungen, die die gesellschaftliche Anerkennung bestimmter Formen von Identitäten einklagen, müssen annehmen, daß die Unterschiede, in deren Namen sie sich einsetzen, für ihr Leben als Individuen grundlegend und essentiell sind. $\ll^{13}$

Die frühen Debatten der 1990er-Jahre umfassen Positionen, die einerseits für eine Absage an ein um eine eindeutige Identität angeordnetes politisches Subjekt stehen (Butler), genauso wie Positionen, die es bis zu einem gewissen Grad als notwendig für das emanzipatorische feministische Projekt erachten, ein gemeinsames Subjekt »Frau« zu setzen (Benhabib). ${ }^{14}$ Nancy Fraser nimmt im "Streit um die Differenz« eine vermittelnde Rolle ein. Im gleichen Band wirft sie sowohl Seyla Benhabib als auch Judith Butler vor, »falsche Gegensätze« hervorzubringen und unnötig zu polarisieren. Fraser plädiert hingegen dafür, einen Weg zu finden, bei dem feministische Politiken von den jeweiligen Vorzügen der beiden Perspektiven profitieren können. ${ }^{15}$ Auch wenn queere Theorien eher auf Seiten Judith Butlers verortet werden, beschäftigen die Kritiken, die Benhabib aber auch Fraser in den 1990er-Jahren eingebracht haben und die mittlerweile vielfach fortgeführt und weitergedacht wurden, queere Theorie und Praxis bis heute (wenn auch zum Teil in einer etwas verschobenen Art und Weise).

\subsection{Interventionen Schwarzer Feminist*innen: Ain't I a Woman?}

Wenn ein Subjekt, eine Geschichte und eine Gesellschaftskritik Teil eines emanzipatorischen feministischen Projekts sind, stellt sich aus der Perspektive Schwarzer Feminist*innen auch die Frage, welches Subjekt, welche Geschichte und welche Gesellschaftskritik dort eingesetzt werden. Oft werden und wurden in feministischen Bewegungen, so die Kritik Schwarzer Feminist*innen, ein bestimmtes weißes Subjekt, eine

11 Ebd., S. 28.

12 Ebd., S. 24.

13 S. Benhabib: Kulturelle Vielfalt und demokratische Gleichheit, S. 24.

14 Die Frage, ob queere Politiken mit dem Anspruch, im Sinne Butlers, das eigene politische Subjekt selbst zur Verhandlung zu stellen, auf eine Geschichte, Gesellschaftskritik und eine »erste Person«, für die Identität von Bedeutung ist, verzichten können, wird auch im Zuge der vorliegenden empirischen Untersuchung wiederaufgenommen werden. 
bestimmte weiße Geschichte und eine bestimmte weiße Gesellschaftskritik als für alle Frauen universell gültig gesetzt. Schon lange vor den Interventionen Judith Butlers haben Schwarze und Feminist*innen of Color darauf hingewiesen, dass diese Universalität fiktional sei und eindeutige Identitätspolitiken mehrfachmarginalisierte Gruppen ausschließen und unsichtbar machen würden. Bereits 1851 stellte die früher versklavte Sojourner Truth auf der Frauenrechtskonvention in Ohio die berühmte Frage »Ain't I a woman?«. Die mehrheitlich weißen Suffragetten wollten damals eigentlich verhindern, dass Truth diese bekannt gewordene Rede hält. Sie hatten Angst, Truth würde von ihrem Thema - dem Frauenwahlrecht - ablenken. In ihrer Rede setzte Sojourner Truth dem Narrativ weißer Männer, Frauen seien zu »schwach «, um an der Politik teilhaben zu können, ihre eigenen Erfahrungen als Sklavin entgegen: »Look at my arm! I have ploughed and planted and gathers into barns, and no man could head me - and ain't I a woman «. ${ }^{16}$ Truth legt in dieser Rede die Lücke zwischen ihren eigenen verkörperten Erfahrungen als afroamerikanische Frau und der eigentlichen Kategorie »Frau « offen und fordert ihren Platz in dieser Kategorie ein. ${ }^{17}$ Erst hundert Jahre nach dieser bekannten Rede gelang es Feminist*innen of Color, wie zum Beispiel dem »Combahee River Collective (CRC)«, Audre Lorde oder Kimberle Crenshaw, nachhaltig auf die Unsichtbarmachung von Mehrfachunterdrückungen in feministischen Politiken, aber auch darüber hinaus aufmerksam zu machen.

\section{Die Kritik der Schwarzen Frauenbewegung}

Wegweisend waren vor allem Schwarze Aktivist*innen, die ausgehend von ihren Erfahrungen in der Bürgerrechtsbewegung und der Frauenbewegung ihre Marginalisierung als Schwarze Frauen zu thematisieren begannen. Bereits 1970 wurde in dem Sammelband »The Black Woman, an Anthology ${ }^{18}{ }^{8}$ ein Text der »SNCC (Student Nonviolent Coordinating Committee) « ${ }^{19}$-Aktivist*in Francis Beal mit dem Titel »Double Jeopardy: To Be Black and Female« veröffentlicht. In diesem Artikel macht Beal auf die doppelte Unterdrückung Schwarzer Frauen in den sozialen Bewegungen, aber auch am Arbeitsmarkt und in der Gesellschaft generell aufmerksam. ${ }^{20}$ Ausgangspunkt des Aufsatzes waren neben Ausschlusserfahrungen in der Frauenbewegung vor allem Marginalisierungen, die aufgrund struktureller und inhaltlicher Veränderungen bei »SNCC « auftraten. Während Frauen bei »SNCC« zu Beginn vergleichsweise »gleichberechtigt« agieren konnten, änderte sich ab Mitte der 1960er-Jahre der Ton in der Gruppe. Diese begann nicht nur, sich von den früheren Prinzipien der Gewaltfreiheit loszusagen und weiße Aktivist*innen aus der Bewegung auszuschließen, sondern stellten auch zunehmend die Rolle von Frauen als "gleichwertige« Aktivist*innen in Frage: »That is, black men began defining the role of black women in the movement. They stated that our

\footnotetext{
16 Sara Ahmed: Feministisch leben! MANIFEST FÜR SPASSVERDERBERINNEN, Münster: Unrast Verlag 2017, S. 120.

17 Ebd.

18 Toni Cade (Hg.): The Black Woman. An Anthology, New York: Simon \& Schuster 1970.

19 »SNCC« war eine bedeutende Organisation in der Bürgerrechtsbewegung.

20 Frances Beal: »Double Jeopardy. To Be Black and Female«, in: Meridians: Feminism, Race, Transnationalism 8 (2008), S. 166-176.
} 
role was a supportive one, others stated that we must become breeders and provide an army; still others stated that we had Kotex or pussy power «. ${ }^{21}$ Als Reaktion auf diese Veränderungen beantragte Francis Beal 1968 die Gründung eines eigenen Komitees bei "SNCC «, das sich mit Sexismus und Rassismus auseinandersetzte. Aus diesem Komitee entstand eine der ersten Schwarzen Frauenorganisationen: Die »Third World Women Alliance (TWWA)«. ${ }^{22}$ Viele Schwarze Männer warfen den Schwarzen Frauengruppen damals vor, sie würden die Schwarze Befreiungsbewegung spalten. ${ }^{23}$ Aber nicht nur der Sexismus in der Bürgerrechtsbewegung stellte ein Problem für die Schwarzen Frauen dar, sondern auch der Rassismus der weißen Frauenbewegung. Beal betont allerdings in ihrem Text »Double Jeopardy« durchaus - basierend auf gemeinsam geteilten Ausbeutungsverhältnissen - die Möglichkeit der Allianz mit weißen Frauen. Sie konstatiert aber auch, dass solche Koalitionen nur möglich seien, wenn weiße Gruppen Rassismus und Imperialismus in den Blick nehmen: »Any white group that does not have an antiimperalist and anti-racist ideology has absolutely nothing in common with the black women's struggle. ${ }^{24}$

Während Beal heute für ihre additive Vorstellung von Unterdrückung kritisiert wird, ${ }^{25}$ thematisierte das "Combahee River Collective (CRC) ${ }^{26}$ als eine der ersten aktivistischen Gruppen die Überschneidung und Überlappung von Unterdrückungssystemen: »The most general statement of our politics at the present time would be that we are actively committed to struggling against racial, sexual, heterosexual, and class oppression and see as our particular task the development of integrated analysis and practice based upon the fact that the major systems of oppression are interlocking. ${ }^{27}$ Das »CRC «-Statement, das 1977 veröffentlicht wurde, gilt als einer der Schlüsseltexte der Intersektionalitätsforschung. Die Gruppe selbst spaltete sich 1975 von der »National Black Female Organisation (NBFO)«ab, weil diese Homosexualität und Klassenverhältnisse zu wenig thematisierten. Ähnlich wie im Fall der »TWWA«, gehen auch die Positionen und Interventionen des »Combahee River Collective« von den Marginalisierungserfahrungen in anderen sozialen Bewegungen aus. ${ }^{28}$

Wie am Beispiel der »Third World Women Alliance« und dem »Combahee River Collective« deutlich wird, begannen insbesondere Schwarze und Aktivist*innen of Color bereits in den 1970er-Jahren, soziale Bewegungen, die eine eindeutige IdentitätsKategorie zum Ausgangspunkt ihrer Politiken machten, kritisch in den Blick zu neh- 
men. Ausgehend von der Erkenntnis, dass diese Bewegungen Schwarze Frauen nicht repräsentierten, gründeten sie eigene Gruppen, um die je eigenen Erfahrungen, die auf sich überlappende Unterdrückungssysteme zurückgehen, zu politisieren. Gleichzeitig blieben zumindest die hier genannten Gruppen aber immer auch offen für Allianzen mit anderen Bewegungen. Ende der 1980er-, Anfang der 1990er-Jahre fanden diese Interventionen Schwarzer Aktivist*innen auch Eingang in akademische Debatten.

\section{Intersektionalität}

Mittlerweile werden die Kritiken an der Überlappung von Unterdrückungssystemen meistens unter dem »Paradigma der Intersektionalität « - wie Katharina Walgenbach es bezeichnet - diskutiert. ${ }^{29}$ Kimberle Crenshaw gilt als die erste, die den Begriff ins akademische Feld eingeführt hat. 1989 ist unter dem Titel »Demarginalizing the Intersection of Race and Sex: A Black Feminist Critique of Antidiscrimination Doctrine, Feminist Theory and Antiracist Politics« der erste Artikel von Crenshaw, der Intersektionalität thematisiert, erschienen. ${ }^{30}$ In diesem Artikel werden verschiedene juristische Fälle beschrieben, in denen die spezifischen Erfahrungen Schwarzer Frauen aufgrund eines »single-axis-Framework" herausfallen. Im ersten Fall beschreibt Crenshaw den Versuch von Schwarzen Frauen, gegen General Motors zu klagen, weil diese keine Schwarzen Frauen angestellt hatten. Sie durften aber nicht als Schwarze Frauen klagen, weil Unterdrückungskategorien nicht beliebig addiert werden könnten, so das juristische Argument. Als Frauen konnten sie nicht klagen, weil weiße Frauen angestellt waren, und als Schwarze konnten sie nicht klagen, weil Schwarze Männer einen solchen Prozess bereits verloren hatten. In einem anderen Fall konnte Moore nicht gegen die Firma Hughes Helicopter klagen, weil sie als Schwarze Frau nicht die Gruppe der Frauen an sich vertreten durfte. In einem dritten Fall durften Schwarze Frauen nicht für die Gruppe der Schwarzen klagen.

Am Beispiel dieser drei juristischen Fälle will Crenshaw aufzeigen, wie Schwarze Frauen in mehrfacher Hinsicht ausgelöscht werden: Sie sind nicht privilegiert genug, um die Gruppe der Frauen oder Schwarzen zu vertreten, dürfen aber auch nicht als Gruppe der Schwarzen Frauen klagen. Gerade in dieser Widersprüchlichkeit manifestiert sich Crenshaw zufolge die Limitierung einer »single-axis«-Konzeptualisierung: »I have stated earlier that the failure to embrace complexities of compoundedness [...] is due to the influence of a way of thinking about discrimination which structures politics so that struggles are categorized as singular issues. Moreover, this structure imports a descriptive and normative view of society that reinforces the status quo! « ${ }^{31}$ Intersektionalität ist demgegenüber ein Konzept, mit dem die Komplexität, der eine »single-axis«-Konzeptualisierung nicht gerecht werden kann, fassbar gemacht werden soll:

Katharina Walgenbach: Intersektionalität-eine Einführung 2012, https.//www.portal-intersektionalität.de vom 25.05.2020.

30 Kimberle Crenshaw: »Demarginalizing the Intersection of Race and Sex: A Black Feminist Critique of Antidiscrimination Doctrine, Feminist Theory and Antiracist Politics«, in: University of Chicago Legal Forum (1989), S. 139-167. 
In einem »Intersektionalen Paradigma« rücken die intersections von verschiedenen Unterdrückungskategorien, aus denen spezifische eigene Marginalisierungserfahrungen hervorgehen können, in den Fokus. Nicht nur in juristischen Fällen, sondern auch in sozialen Bewegungen - das wird an den zu Beginn angeführten Beispielen der »TWWA« und des »CRC« deutlich - läuft der Fokus auf »single-axis«-Politiken Gefahr, Erfahrungen, die aus der intersection mehrerer Unterdrückungserfahrungen resultieren, auszulöschen.

Die Antworten von Frauen of Color auf intersektionale Kritiken an einfachen Identitäts-Kategorien als Ausgangspunkt politischer Bewegungen fallen allerdings unterschiedlich aus. Crenshaw selbst betont, dass Kategorien sozial wirkmächtig seien und es deswegen notwendig sei, strategisch auf sie zurückzugreifen: »But to say that a category such as race or gender is socially constructed is not to say that that category has no significance in our world. $\aleph^{32}$ Ihrer Meinung nach sind es auch nicht die Kategorien an sich, die problematisch sind, sondern die Bedeutungen, die an sie geknüpft sind: "And this project's most pressing problem, in many if not most cases, is not the existence of the categories, but rather the particular values attached to them and the way those values foster and create social hierarchies. ${ }^{33}$ Es geht ihr also nicht darum, sich von den Kategorien zu befreien, sondern die Kategorien von den Bedeutungen $\mathrm{zu}$ befreien, die an sie geknüpft sind. Auch das oben genannte »Combahee River Collective« kann und will nicht auf Identitätspolitiken verzichten: »This focusing upon our own oppression is embodied in the concept of identity politics. We believe that the most profound and potentially most radical politics come directly out of our own identity, as opposed to working to end somebody else's oppression. « ${ }^{34} \mathrm{Ja} \mathrm{zu}$ sagen zu einer Politik, die die eigene mehrfach-unterdrückte Identität zum Ausgangspunkt macht, bedeutete für das »CRC« nicht länger, nur für die Befreiung anderer, sondern endlich für die eigene Befreiung kämpfen zu können. Im »CRC«-Statement wird aber auch Kritik an jeglicher Form des Separatismus geübt. Gemeinsame Politiken mit Bewegungen, die nur eine Achse der Unterdrückung denken, bleiben für das »CRC« weiterhin denkbar: »We struggle together with Black men against racism, while we also struggle with Black men about sexism. ${ }^{35}$ Crenshaws Position und die des »CRC« sind beide weit davon entfernt, Identitätspolitiken eine Absage zu erteilen. Sie fordern aber andere Identitätspolitiken. Solche, die »strategisch" sind, und solche, die »the interlocking system of oppression " in den Blick nehmen, aber trotzdem weiterhin solidarisch mit anderen Bewegungen bleiben, ohne Differenzen zu glätten. ${ }^{36}$

K. Crenshaw: Mapping the Margins: Intersectionality, Identity Politics, and Violence against Women of Color, S. 1296.

Ebd.

34 The Combahee River Collective: A Black Feminist Statement, S. $273 \mathrm{f}$.

35 Ebd., S. 274.

36 Neben diesen beiden Positionen gelten auch die Sammelbände »This Bridge Called My Back« von Cloria Anzaldúa und Cherrie Moraga (1981) und »All the Women Are White, All the Blacks Are Men, But Some of Us Are Brave: Black Women's Studies«, herausgegeben von Cloria T. Hull, Patricia Bell Scott und Barbara Smith (1982), sowie Theoretiker*innen wie beispielsweise bell hooks, Audre Lorde und Angela Davis als wegweisend für Intersektionalität. Vgl. K. Walgenbach: Intersektionalität. 
Andere Schwarze Feminist*innen kritisieren hingegen Formierungen sich separierender feministischer Gruppen. Diese würden zum einen die Möglichkeit aufgeben, eine andere feministische Ideologie $\mathrm{zu}$ formulieren, zum anderen würden sie die Bedingungen der eigenen Unterdrückung affirmieren:

»By creating segregated feminist groups, they both endorsed and perpetuated the very racism they were supposedly attacking. They did not provide a critical evaluation of the women's movement and offer to all women a feminist ideology uncorrupted by racism or the opportunistic desires of individual groups. Instead, as colonized people have done for centuries, they accepted the terms imposed upon them by the dominant group [...]. $\ll^{37}$

Ähnlich wie die angeführten Debatten fallen auch aktuelle bewegungspolitische Antworten auf intersektionale Kritiken, genauso wie akademische intersektionale Debatten, sehr unterschiedlich aus. Mitunter sind sie mittlerweile dem Vorwurf ausgesetzt, die Kategorie Klasse vernachlässigt $\mathrm{zu}$ haben beziehungsweise nicht einmal dazu in der Lage zu sein, Klassenverhältnisse zu artikulieren.

Tove Soiland beispielsweise spricht von einer Unvereinbarkeit von Klassenpolitiken und intersektionalen Politiken, die sie als Identitätspolitiken bezeichnet. Sie sieht zumindest in der Art, wie gegenwärtig intersektionale Antidiskriminierungspolitiken sich äußern, keine Möglichkeit, eine Interferenz mit der gesellschaftstheoretischen Kategorie Klasse denkbar zu machen. Soiland bezeichnet Identitäts-Kategorien, als Benachteiligungs-Kategorien, die zwar für Antidiskriminierungspolitiken sinnvoll seien, aber nicht mit gesellschaftstheoretischen Artikulationen wie Klassenverhältnissen interferierend gedacht werden könnten. Solche Antidiskriminierungspolitiken hätten ihr zufolge die »Befreiung aus der Kategorie« als einziges Ziel und könnten nicht nach den Mechanismen der Unterdrückung dieser Kategorien fragen. ${ }^{38}$ Wer Intersektionalität per se mit Politiken gleichsetzt, die mitunter wirklich nicht dazu in der Lage sind, über Klassenverhältnisse zu sprechen, vergisst allerdings, dass Klasse von Anfang an ein zentrales Thema intersektionaler Kritiken war. Viele Schwarze Frauen waren Arbeiter*innen. Entsprechend standen Klassenverhältnisse, wie beispielsweise die Positionen von Beal oder der »CRC« verdeutlichen, von Anfang an im Vordergrund. So schreibt das »CRC « in seinem Statement: »We need to articulate the real class situation of persons who are not merely raceless, sexless workers, but for whom racial and sexual oppression are significant determinants in their working/economic lives. « ${ }^{39}$

Auch intersektionale Debatten geben unterschiedliche Antworten auf die Frage, wie mit der Kritik an einfachen und eindeutigen Identitätspolitiken umzugehen ist. Sie sind (in ihrer Unterschiedlichkeit) mittlerweile, genauso wie postkoloniale Perspektiven, wichtige Bestandteile queerer Theorie und Politik. ${ }^{40}$

\footnotetext{
37 bell hooks: Ain’t I a Woman. Black Women and Feminism, New York: Routledge 1981, S. 151.

38 T. Soiland: Die Verhältnisse gingen und die Kategorien kamen.

39 The Combahee River Collective: A Black Feminist Statement, S. 274.

40 Die Rolle intersektionaler und postkolonialer Perspektiven für aktuelle queere Politiken wird in der empirischen Untersuchung der vorliegenden Arbeit noch thematisiert werden.
} 


\subsection{Eine postkolonial-feministische Perspektive: Can the Subaltern Speak?}

Postkoloniale Perspektiven legen den Fokus auf die »Produktion und Unterwerfung des kolonisierten Subjekts « ${ }^{41}$ Sie zeigen auf, inwiefern auch in einem postkolonialen Zeitalter das unzivilisierte, unmoderne, ehemalig kolonisierte Subjekt in Abgrenzung zum zivilisierten, modernen westlichen Subjekt produziert wird. Gayatri Chakravorty Spivak gilt als eine der prominentesten Theoretiker*innen, die eine feministische Perspektive mit einer postkolonialen Perspektive verknüpft. ${ }^{42}$ Sie zeigt unter anderem auf, wie »Gender zum Alibi kolonialer Herrschaft« werden konnte, indem eine unmoderne Geschlechterpolitik zum Beweis für die Unzivilisiertheit der kolonialisierten Nationen gemacht wurde. ${ }^{43}$

In ihrem bekannten Aufsatz »Can the Subaltern Speak? « lenkt sie ausgehend vom Konzept der Subalternität den Blick auf diejenigen Gruppen, die in gängigen (post-)kolonialistischen Subjektproduktionen nicht mitgedacht werden. Der 1988 zum ersten Mal erschienene Text gilt als der meistgelesene und -rezipierte Aufsatz der postkolonialen Theorie. ${ }^{44}$ Spivak selbst wollte nicht, dass die erste Version ihres Aufsatzes in den 1996 erschienenen »Spivak Reader « aufgenommen wird. Stattdessen veröffentlichte sie zunächst ein Interview, in dem sie angibt, dass das Konzept der Subalternität vielfach inflationär verwendet und missverstanden rezipiert worden sei: »Ich glaube das Wort ssubaltern verliert hier seine Definitionskraft, weil es zu einer Art Schlagwort für jede beliebige Gruppe geworden ist, die etwas will, was sie nicht hat. ${ }^{45}$ Erst elf Jahre nach dem ersten Erscheinen des Aufsatzes - 1999 - wird eine überarbeitete Fassung des Textes im Band »Kritik der postkolonialen Vernunft« veröffentlicht. Diese Fassung beschreibt Spivak im Vergleich zur ersten Version des Aufsatzes als viel geradliniger. Die erste Version spiegle dementgegen den Kampf wider, den sie damals während des Schreibens mit sich ausgetragen habe. Die 1988 erschienene erste Version - die 2008 auch auf Deutsch veröffentlicht wurde - ist der primäre Bezugspunkt der folgenden Darstellung. Der Aufsatz ist in mehrere Abschnitte unterteilt: Im ersten Abschnitt wirft Spivak Foucault und Deleuze mit Marx vor, ein ungeteiltes souveränes Subjekt vorauszusetzen. Im zweiten Abschnitt kritisiert sie den Versuch der marxistisch orientierten »Subaltern Studies Group«, eine »Geschichte von unten« zu schreiben, indem sie sich poststrukturalistischer Denkwerkzeuge bedient. Im dritten Teil nimmt Spivak Bezug auf Derrida, der ihr zufolge im Gegensatz zu Foucault nicht in einer eurozentristischen

Nikita Dhawan: »Politische Theorie der Subalternität. Gayatri Chakravorty Spivak«, in: André Brodocz/Gary Schaal (Hg.), Politische Theorien der Cegenwart III. Eine Einführung, Opladen: Barbara Budrich Verlag 2016, S. 169-196, hier S. 170.

42 Neben Spivak ist insbesondere auch Chandra Talpade Mohanty, vor allem ihr Aufsatz »Under Western Eyes (1984), stark rezipiert in den deutschsprachigen Gender Studies.

44 Ebd., S. 178.

45 Gayatri C. Spivak: »Ein Gespräch über Subalternität«, in: Boris Buden/Jens Kastner/Oliver Marchart et al. (Hg.), Can the subaltern Speak? Postkolonialität und subalterne Artikulation, Wien: Turia + Kant Verlag 2008, S. 119-148, hier S. 123. 
Perspektive verhaftet bleibe. ${ }^{46}$ Im vierten und letzten Abschnitt widmet sie sich dann der weiblichen Subalternen und zeigt auf, inwiefern diese nicht sprechen kann.

\section{Die Massen können für sich selbst sprechen}

$\mathrm{Zu}$ Beginn des Aufsatzes setzt Spivak sich kritisch mit dem poststrukturalistischen Denken von Foucault und Deleuze auseinander. Spivaks Kritik an Foucault und Deleuze bezieht sich auf ein Gespräch zwischen den beiden Theoretikern: ${ }^{47}$ "Ich habe diesen freundlichen Austausch zwischen zwei aktivistischen Geschichtsphilosophen ausgewählt, weil er die Opposition zwischen autoritativer theoretischer Produktion und der ungeschützten Praxis des Gesprächs auflöst und so einen Blick auf die Wege der Ideologie ermöglicht. ${ }^{48}$ In einer Fußnote, die sie gleich im Anschluss an die Begründung ihrer Textauswahl setzt, erklärt Spivak, dass sie die Übersetzung des Gesprächs aus dem Französischen ins Englische zum Teil modifiziert habe: "Ich habe die englische Version dieses Textes, wie auch anderer englischsprachiger Übersetzungen, modifiziert, soweit Treue zum Original dies zu verlangen schien. «" ${ }^{49}$ Es erscheint befremdlich, dass Spivak ihre starke Kritik an zwei bekannten französischen Theoretikern nicht auf deren Hauptwerke, sondern auf ein Gespräch bezieht und gleichzeitig im Namen der »Treue zum Original« die Übersetzung modifiziert, so dass sie vermeintlich sogar Spivaks eigene Argumentation festigt. ${ }^{50}$ Vielleicht verweisen diese beiden Aspekte, die gleich zu Beginn ihres Aufsatzes auffallen, aber auch auf ein Schreiben, das insofern in der postkolonialen Theorie verortet ist, als es beispielsweise durch absichtliches "missreading « westliche Wissens-Regime aufzubrechen versucht.

Was kritisiert Spivak nun aber an den Aussagen von Foucault und Deleuze aus diesem Gespräch? Zum einen wirft sie ihnen vor, dass sie ungeteilte souveräne politische Subjekte voraussetzen; zum anderen, dass sie unbewusst eurozentrisch sind, weil sie internationale Arbeitsteilung ignorieren und ihren Blick lediglich auf Mikropolitiken legen. Warum aber ist Spivak der Meinung, dass gerade Theoretiker wie Foucault und Deleuze die »Dominierten als klassisch humanistische Subjekte konstruieren? ${ }^{51}$ Oder auch mit ihren eigenen Worten: »Warum sollte solchen Schließungen ausgerechnet im

46 Dieser sehr kurze Abschnitt wird in den folgenden Ausführungen nicht näher erläutert.

47 Das Gespräch zwischen Foucault und Deleuze - »Intellektuelle und Macht «-, auf das Spivak sich bezieht, fand anlässlich der Gefängnisaufstände in Frankreich statt, die beide Theoretiker unterstützt haben.

48 Gayatri C. Spivak: „Can the Subaltern Speak?«, in: Boris Buden/Jens Kastner/Oliver Marchart et al. (Hg.), Can the subaltern speak? Postkolonialität und subalterne Artikulation, Wien: Turia + Kant Verlag 2008, S. 17-118, hier S. 21.

49 Ebd., S. 106.

50 So ändert sie beispielsweise folgende Aussage aus der offiziellen englischen Übersetzung »We cannot shut out the scream of Reich; no the masses were not deceived; at a particular moment they actually desired a facist regime« in »We must accept the scream of Reich [...]«. Gayatri C. Spivak: »Can the subaltern Speak?«, in: Cary Nelson/Lawrence Grossberg (Hg.), Marxism and the Interpretation of Culture, Urbana, Chicago: University of Illinois Press 1988, S. 271-333, hier S. 274. Gleichzeitig muss aber hinzugefügt werden, dass sie beispielsweise mit ihrer Übersetzung der Grammatologie von Derrida ihre Fähigkeiten als Übersetzer*in unter Beweis gestellt hat. 
Falle jener Intellektuellen zugestimmt werden, die unsere besten Propheten der Heterogenität und des/der Anderen sind «? ${ }^{52}$ Spivak wirft Foucault und Deleuze vor, die Ideologie zu ignorieren, wenn sie in ihrem Gespräch nicht zwischen »Interesse« und "Begehren« unterscheiden. Folgende Aussage von Deleuze ${ }^{53}$ legt Spivak zufolge eine solche mangelnde Differenzierung nahe: »We never desire against our interests, because interests always follows and finds itself where desire has placed it. $"{ }^{54}$ Sie manifestiere sich aber auch in der Aussage von Deleuze und Foucault, dass die »Massen für sich selbst sprechen können « und es entsprechend die Aufgabe der Intellektuellen sei, ihnen den Raum dazu zu geben: »the masses know perfectly well clearly, [...] they know far better than the intellectual and they certainly say it very well. ${ }^{55}$ In einer solchen Vorstellung, der zufolge das Begehren immer dem Interesse folge, wird laut Spivak das Feld der Ideologie vernachlässigt und das unterdrückte Subjekt, das für sich selbst sprechen kann, aufgewertet. ${ }^{56}$ Foucault und Deleuze würden voraussetzen, dass Subjekte, die rein formal der gleichen Klasse angehören - und somit das gleiche Klasseninteresse haben -, sich folglich auch als ein gemeinsames politisches Subjekt mit gleichen Interessen verstehen, das um die eigene Unterdrückung weiß, und entsprechend für sich selbst sprechen können.

Deleuze ruft im Zuge dieser Argumentation ein post-repräsentationales Zeitalter aus: »Es gibt keine Repräsentation mehr, es gibt nur noch Aktion. ${ }^{57}$ In dieser Aussage wird Spivak zufolge nicht zwischen den zwei Bedeutungen von Repräsentation unterschieden: "Repräsentation als `sprechen für $<$, wie in der Politik, und Repräsentation als $>$ Re-präsentation<, als >Dar-stellungく bzw. >Vorstellungく, wie in der Kunst oder Philosophie. ${ }^{58}$ Spivak kritisiert diese mangelnde Unterscheidung, indem sie den Aussagen von Foucault und Deleuze Passagen aus dem »Achtzehnten Brumaire des Louis Bonaparte« von Karl Marx entgegensetzt. Marx gehe im Gegensatz zu Foucault und Deleuze von einem disparaten und dislozierten Subjekt aus: "Sowohl auf ökonomischen Gebiet (Kapitalist), als auch auf politischem Gebiet (weltgeschichtlicher Akteur) sieht sich Marx genötigt, Modelle eines geteilten und dislozierten Subjekts zu entwerfen, dessen Teile keinerlei Zusammenhang und Kohärenz aufweisen. ${ }^{59}$ Marx spricht im »Achtzehnten Brumaire des Louis Bonaparte« von dem fehlenden Klassenbewusstsein der französischen Parzellenbauern, die durch einen Bevollmächtigten aus der Mittelschicht ver-

C. C. Spivak: Can the Subaltern Speak?, S. 23.

Diese Aussage schreibt sie 1988 noch »den Philosophen«, also Foucault und Deleuze, zu, obwohl sie nur von Deleuze stammt. In der zehn Jahre später erschienenen Version ist sie diesbezüglich genauer.

G. C. Spivak: Can the subaltern Speak?, S. 274.

Ebd. Diese Aussage ist vielleicht auch im Kontext seiner Vorlesung »Theorien und Institutionen der Strafe« zu verstehen, die zeitnah zu dem Gespräch gehalten wurde. Hier beschreibt Foucault nämlich den Intellektuellen als denjenigen, der das für die Macht (die Zentralisierung der Staatsmacht) wichtige Über-Wissen gewinnt, das der Entstehung eines »Volkswissens« zuwiderläuft. François Ewald/Alessandro Fontana/Bernard E. Harcourt et al. (Hg.): Theorien und Institutionen der Strafe. Vorlesungen am Collège de France 1971-1972, Berlin: Suhrkamp Verlag 2017, S. 275.

G. C. Spivak: Can the Subaltern Speak?, S. 25.

Ebd., S. 28.

Ebd., S. 29.

Ebd., S. 31. 
treten (repräsentiert) werden: »Das (mangelnde kollektive) Bewusstsein der Klasse von Parzellenbauern findet ihren >Träger $<$ in einem >Repräsentanten $<$, einem >Vertreter $<$, der in jemandes anderen Interesse zu arbeiten scheint. Das Wort >Repräsentant $<$ leitet sich hier nicht von >darstellen ab. $^{60}$

Was Spivak mit der Unterscheidung zwischen Repräsentation als »sprechen für« einerseits und Repräsentation als "sprechen von « andererseits aufzeigen will, ist, dass es immer eine Lücke "Zwischen ästhetischer und politischer Repräsentation " gibt. ${ }^{61}$ Diese Lücke ist zwangsläufig größer, wenn es um die politische Repräsentation der Subalternen geht. Ein authentisches Sprechen, wie Foucault und Deleuze es fordern, ist ihr zufolge nicht möglich.

\section{Eine Geschichte von unten schreiben}

Im zweiten Abschnitt des Aufsatzes stellt Spivak die »Subaltern Studies Group« von Ranajit Gua vor, deren Ziel es ist, mit klassisch marxistischen Methoden eine Geschichte »von unten $«$ zu schreiben. ${ }^{62}$ Die »Subaltern Studies Group« übernahm den Begriff der Subalternität von Antonio Gramsci, weil sie zwischen den Arbeiter*innen in Süditalien $^{63}$ und der Landbevölkerung in Indien Parallelen erkannte. Gua »definiert Subalternität als einen Raum, der innerhalb eines kolonialisierten Territoriums von allen Mobilitätsformen abgeschnitten ist. ${ }^{64}$ Subalternität ist eine Position, die sich aus der Differenz zu den Eliten und all denjenigen ergibt, die sich eben innerhalb dieser Linien der Subalternität bewegen. ${ }^{65}$ Es handelt sich also nicht um einen Identitätsbegriff, sondern um »eine Position in Differenz«. ${ }^{66}$ Während Gua den Begriff der Subalternen nur für Kollektive verwendet, kann der Begriff bei Spivak auch einzelne Personen bezeichnen. Für Spivak beinhaltet die Definition der Subalternität an sich eine Unfähigkeit, Sprechakte zu setzen. So schreibt sie: »Niemals sehen wir die reinen Subalternen. ${ }^{67}$ In dem Moment, in dem wir sie sehen, sind sie keine Subalternen mehr. In diesem Sinne kann subalterner Widerstand als ein Versuch gesehen werden, sich selbst zu repräsentieren, »und zwar nicht entlang der Linien, die von den offiziellen institutionellen Repräsentationsstrukturen vorgegeben werden «. ${ }^{68}$

Nachdem Spivak nun zuvor gewissermaßen mit Marx Kritik an Foucault und Deleuze geübt hat, kann dieser zweite Abschnitt als poststrukturalistische und auch feministische Kritik an dem marxistischen Versuch der "Subaltern Studies Group», die Geschichte neu zu schreiben, gelesen werden. ${ }^{69}$ Eine Geschichte von unten zu schreiben, läuft Spivak zufolge Gefahr, das heterogene subalterne Subjekt zu homogenisie-

60 Ebd.

61 N. Dhawan: Politische Theorie der Subalternität, S. 180.

62 G. C. Spivak: Can the Subaltern Speak?, S. 48.

63 Gramsci bezeichnete die Arbeiter*innen in Süditalien als die Subalternen.

64 M. d. M. Castro Varela/N. Dhawan: Postkoloniale Theorie, S. 187.

65 G. C. Spivak: Can the Subaltern Speak?, S. 49.

66 M. d. M. Castro Varela/N. Dhawan: Postkoloniale Theorie, S. 187.

67 G. C. Spivak: Ein Gespräch über Subalternität, S. $121 \mathrm{f}$.

68 Ebd., S. $144 \mathrm{f}$.

69 N. Dhawan: Politische Theorie der Subalternität, S. $176 f$. 
ren. ${ }^{70}$ Aus feministischer Perspektive kritisiert Spivak zudem, dass Frauen in diesem Versuch, die Geschichte neu zu schreiben, doppelt ausgelöscht werden: »If in the context of colonial production, the subaltern has no history and cannot speak, the subaltern as female is even more deeply in shadow. ${ }^{71}$ Die Geschichte der Subalternen könne niemals vollständig erfasst werden. ${ }^{72}$ Die Idee einer vollständigen Erfassung subalternen Bewusstseins führe zu einer »romantisierenden Repräsentation von Subalternen als einem Widerstandssubjekt mit klaren Intentionen $« .{ }^{73}$ Der Versuch, eine authentische Stimme einzufangen, stabilisiere demzufolge eine westliche Vorstellung, nach der Sprechen Subjektivität ausdrücke. Trotzdem braucht es, so Spivak, diese Formen der Repräsentation. Sie sind gleichzeitig notwendig und problematisch. Obwohl das subalterne Bewusstsein Spivak zufolge immer etwas Fiktionales ist, hat es doch einen hohen strategischen Wert, ermöglicht es beispielsweise dennoch, die dominante Geschichtsschreibung einer fundamentalen Kritik zu unterwerfen. ${ }^{74}$

\section{Die Subalterne kann nicht sprechen}

Im letzten Abschnitt legt Spivak ihren Fokus auf den »vergeschlechtlichten Ort subalterner Frauen «. ${ }^{75}$ Am Beispiel der Witwenverbrennung und eines Selbstmordes zeigt sie auf, dass Frauen in doppelter Hinsicht ausradiert werden. Sati ist eine Praxis der Witwenverbrennung und eine der wenigen Möglichkeiten des anerkannten Suizids für Frauen in Indien: ${ }^{76}$ Witwen folgen ihren verstorbenen Ehemännern in den Tod, indem sie sich verbrennen lassen. ${ }^{77}$ Diese Praxis kommt eher selten vor und mitunter spielen ökonomische Interessen der Verwandtschaft eine Rolle. Die Witwe, die verbrannt wird, würde sonst den Besitz ihres Ehemanns erben. ${ }^{78}$ Spivak beschäftigt sich sowohl mit vedischen und hindu-Texten zur Witwenverbrennung als auch mit den Diskursen des britischen Empires. ${ }^{79}$ In diesen Texten zeigen sich ihr zufolge zwei dominante Ideologien, die sich einerseits entgegenstehen, andererseits gegenseitig legitimieren: Auf der einen Seite steht die Ideologie der Briten, die die Witwenverbrennung kriminalisieren und sich selbst als Retter der Frauen darstellen. Es handelt sich mit Spivaks bekannten Worten um den Topos: »Weiße Männer retten braune Frauen vor braunen Männern. « 80 Spivak merkt in diesem Zusammenhang ironisch an, dass die Briten nicht einmal die Namen der Frauen, die sie zu retten vorgeben, richtig buchstabieren könnten. ${ }^{81}$ Dem gegenüber stehen die Ideologien der einheimischen Männer, die behaupten, dass die

G. C. Spivak: Can the subaltern Speak?, S. 284.

Ebd., S. 287.

G. C. Spivak: Can the Subaltern Speak?, S. 49.

Ebd.

M. d. M. Castro Varela/N. Dhawan: Postkoloniale Theorie, S. 191.

N. Dhawan: Politische Theorie der Subalternität, S. 180.

C. C. Spivak: Can the Subaltern Speak?, S. 93.

Ebd., S. 80.

Ebd., S. 88.

N. Dhawan: Politische Theorie der Subalternität, S. 180.

C. C. Spivak: Can the Subaltern Speak?, S. 81.

Ebd., S. 100. 
Frauen sterben wollen, weil sie gute Ehefrauen sind. ${ }^{82}$ Die Frauen werden zwischen diesen zwei dominanten Ideologien zerrieben: »Beide Male spricht das Patriarchat (koloniales und einheimisches) für die subalterne Frau, während man an keiner Stelle auf die Stimme der Frau selbst trifft. $\ll^{83}$ Es liegt auch nicht im Interesse der jeweiligen Gruppen, die Frauen zu ermächtigen, vielmehr sind die Frauenkörper Schauplatz für ganz andere Kämpfe. Während die Kolonisatoren im Namen zivilisatorischer Errungenschaften wie Geschlechtergerechtigkeit ihre imperialistischen Politiken legitimieren, versuchen die kolonisierten Männer ihre Identität zu bewahren, indem sie ihre Frauen vor der Verwestlichung schützen. ${ }^{84}$

Auch im zweiten Beispiel, das Spivak in ihrem Aufsatz "Can the Subaltern Speak?« anführt, wird deutlich, inwiefern die Subalterne nicht sprechen kann. Spivak erzählt die Geschichte von Bhuvaneswari Bhaduri, einer Aktivistin, die Selbstmord begeht, weil sie den Auftrag, ein Attentat auszuführen, nicht durchführen kann und will. Von Spivaks Familie, darunter auch Intellektuelle mit ähnlichem theoretischem Hintergrund wie Spivak, wurde der Selbstmord immer nur als Folge einer ungewollten Schwangerschaft abgetan. Dabei führte Bhuvaneswari Bhaduri den Selbstmord entgegen dessen Regeln, während sie ihre Menstruation hatte, durch, um so den Akt des Selbstmordes neu in ihren Körper einzuschreiben. Bhuvaneswari Bhaduri konnte, so Spivak, nicht sprechen, weil niemand diese Neueinschreibung des Selbstmordes entziffert hat: »Die Subalterne kann nicht sprechen, das meint also, dass sogar dann, wenn die Subalterne eine Anstrengung bis zum Tode unternimmt, um zu sprechen, dass sie sogar dann nicht fähig ist, sich Gehör zu verschaffen - und Sprechen und Hören machen den Sprechakt erst vollständig. ${ }^{85}$ Mit ihrer berühmt gewordenen Aussage »die Subalterne kann nicht sprechen ${ }^{86}$ meint Spivak den gesamten Sprechakt, der auch das »Hören « mit einschließt.

Ein Aspekt, der in Bezug auf das Beispiel Bhuvaneswari Bhaduri im Anschluss an die erste Version des Aufsatzes heftig diskutiert wurde, ist, dass diese nicht im engeren Sinne eine Subalterne war, weil sie der gehobenen Mittelschicht angehörte. In der zehn Jahre nach der ersten Veröffentlichung erschienenen zweiten Ausgabe des Textes »Can the Subaltern Speak?« antwortet Spivak auf diese Kritik: »Was ich in diesem Kapitel unter anderem wohl darlegen wollte, ist, dass das Abfangen des Anspruches auf Subalternität durch die Frau anhand ihres durch verschiedenartige Umstände verursachten Verstummens über strenge Definitionsgrenzen hinweg festgemacht werden kann. ${ }^{87}$ Subalternität bedeutet für Spivak, nicht sprechen zu können, weil Subalterne von jeglichen Möglichkeiten, sich zu repräsentieren, abgeschnitten sind. Spivak entgegnet - ebenfalls in der neuen Auflage - der Kritik, dass Bhuvaneswari Bhaduri doch

82 Ebd., S. 92.

83 N. Dhawan: Politische Theorie der Subalternität, S. 181.

84 María d. M. Castro Varela/Nikita Dhawan: »Die Migrantin retten!?«, in: Österreichische Zeitschrift für Soziologie 41 (2016), S. 13-28, hier S. 19.

85 G. C. Spivak: Ein Cespräch über Subalternität, S. 127.

86 Gayatri C. Spivak: Can the Subaltern Speak?, Wien: Turia + Kant Verlag 2008, S. 106.

87 Gayatri C. Spivak: »Geschichte«, in: Andreas Nehring/Doris Feldmann/Barbara Gabel Cunningham et al. (Hg.), Kritik der postkolonialen Vernunft. Hin zu einer Geschichte der verrinnenden Gegenwart, Stuttgart: Kohlhammer Verlag 2014, S. 203-306, hier S. 304. 
sprechen konnte, weil Spivak selbst es entziffern konnte: Sprechen können heiße nicht, dass etwas viele Jahre später »akademisch entschlüsselt « werden kann. ${ }^{88}$ Die Subalterne könne nicht sprechen, weil sie, obwohl sie bis zum Tode versucht, zu sprechen, nicht gehört wird. Sie könne aber sehr wohl von einer aufmerksamen Kritiker*in/Intellektuellen gelesen werden. Spivak sieht es als wichtige Verantwortung der Intellektuellen und Feminist"innen, ebendiese blinden Stellen der Subalternität zu entziffern. Voraussetzung sei allerdings, dass die Intellektuellen zuallererst ihre eigene »Komplizenschaft in diesem Verstummen« anerkennen. Diese ethische Verantwortung, die Spivak den Intellektuellen zuschreibt, kann auch als »letzte Pointe zu Foucault und Deleuze gesehen werden «, die gerade fordern, dass die Intellektuellen die Massen für sich selbst sprechen lassen. ${ }^{89}$

Spivak wie auch andere postkoloniale Theoretiker*innen kritisieren mit ihren Interventionen ein westliches Wissensregime, das zumeist internationale Arbeitsteilung ignoriert, den Fokus auf Mikropolitiken im globalen Norden legt und so auch eine Kompliz*innenschaft mit dem Verstummen der Subalternen eingeht. Während die bisher vorgestellten Debatten das den Bedingungen der politischen Repräsentation unterworfene politische Subjekt danach befragten, wer aus dieser Repräsentation ausgeschlossen ist und ob dieses Subjekt den Bedingungen der eigenen Unterwerfung entkommen kann, legt Spivak den Blick auf diejenigen, die von jeglicher Möglichkeit politischer Repräsentation ausgeschlossen sind. Für queere Politiken und Theorien spielen postkoloniale Interventionen, wie sie u.a. von Spivak vorgetragen werden, spätestens seit dem Aufkommen der Homonationalismus-Kritik eine wichtige Rolle. Denn auch queere Politiken laufen, wie im Folgenden ausgeführt wird, Gefahr, zum »Alibi« neokolonialer Politiken zu werden.

\subsection{Queere Auseinandersetzungen: Von der Heteronormativität zum Homonationalismus}

Im akademischen Bereich wird die »begriffliche Geburtsstunde« von »queer« zumeist mit der Literaturwissenschaftler* in Teresa de Lauretis in Verbindung gebracht, die den Begriff auf einer Konferenz 1989 in Santa Cruz/California erstmals einbrachte. ${ }^{90}$ "Queer Nation« hingegen gilt als erste aktivistische Gruppe, die sich den Begriff »queer« 1990 als kollektive Selbstbezeichnung aneignete. ${ }^{91}$ Gloria Anzaldúa verwendete - und das wird in solchen Erzählungen häufig vergessen - »queer« noch vor Teresa de Lauretis und "Queer Nation« in ihrem 1987 erschienenen Buch »Borderlands/La Frontera: The New Mestiza«. Dort versteht sie »queer« als einen Begriff, der über Differenzen hinweg verbindet:

88 Ebd., S. 305.

89 G. C. Spivak: Ein Cespräch über Subalternität, S. 144.

90 Nina Degele: »Heteronormativität entselbstverständlichen: Zum verunsichernden Potenzial von Queer Studies«, in: Freiburger FrauenStudien: Zeitschrift für interdisziplinäre Frauenforschung 11 (2005), S. 15-39., hier S. 17.

91 Meg-John Barker/Julia Scheele: Queer. Eine illustrierte Geschichte, Münster: Unrast Verlag 2018, S. 14. 
»Being the supreme crossers of cultures, homosexuals have strong bonds with the queer white, Black, Asian, Native American, Latino, and with the queer in Italy, Australia and the rest of the planet. We come from all colors, all classes, all races, all time periods. Our role is to link people with each other - the Blacks with the Jews with Indians with Asians with whites with extraterrestrials. ${ }^{92}$

Anzaldúa ist es auch, die einige Jahre später kritisiert, dass vorwiegend »weiße Mittelklasse-Lesben « queere Theorien produzieren und bestimmen, was und wen der Begriff bezeichnet und welches queere Wissen hergestellt wird. ${ }^{93}$ Es gibt aber auch Stimmen, die queere Erzählungen, denen zufolge Feminist*innen of Color als »Geburtshelfer*innen« der "queeren Nicht-Identität« gelten, als gewaltsame Aneignung kritisieren. "Queer« mit »Nicht-Identität«, also dem Tod des Subjekts, gleichsetzend, betont beispielsweise Jinthana Haritaworn, dass es bei den sogenannten »Geburtshelfer"innen« um etwas anders ging:

»Wie Paula Moya (1997), Vivien Ng (1997) und andere zeitgenössische Feministinnen of Colour zeigen, waren diese [Audre Lorde und Cloria Anzaldúa] weit davon entfernt, das ethnisierte und vergeschlechtlichte Subjekt zu ermorden. Ganz im Cegenteil beton(t)en sie, wie der berühmte Titel This Bridge Called My Back (Moraga/Anzaldúa 1981) ausdrückt, die Notwendigkeit von Positionalität für Koalitionen zwischen Frauen und Männern verschiedenster ethnischer, sexueller und Klassenhintergründe. «94

Eine Aneignung der Theorien von Feminist"innen of Color ermögliche es, so ein Kritikpunkt von Haritaworn, durch einen Akt des Einschlusses lediglich, das "weiße queere Gewissen « rein zu halten, während in der Theorieproduktion gleichzeitig von Queers of Color abstrahiert werde. ${ }^{95}$ Nicht nur die Rolle der Theorien von Feminist*innen of Color - deren Interventionen in den vorherigen Abschnitten schon dargelegt wurden -, sondern auch die Rolle Judith Butlers in der Geschichte queerer Theorien ist, wenn auch auf eine andere Art und Weise, umstritten. Auch die Tatsache, dass Judith Butler meist am Anfang einer Geschichte queerer Theorieproduktion zu finden ist, laufe Gefahr, eine zu vereindeutigte queere Geschichte zu erzählen. ${ }^{96}$ Gleichzeitig sei ihr Werk zentral für queere Theorien und es sei beinahe unmöglich geworden, von »queer« zu sprechen, ohne ihren Namen zu nennen. ${ }^{97}$

Butlers 1990 auf Englisch und 1991 auf Deutsch erschienenes Werk »Das Unbehagen der Geschlechter« löste nicht nur kontroverse Debatten in Bezug auf die Frage nach dem politischen Subjekt »Frau« aus, sondern Butler trieb mit der »Dekonstruktion« von

92 Gloria Anzaldúa: Borderlands/La Frontera. The New Mestiza, San Francisco: Aunt Lute Books 1999, S. $106 \mathrm{f}$.

93 Brigitte Bargetz/Gundula Ludwig: »Bausteine einer queerfeministischen politischen Theorie: eine Einleitung«, in: Femina Politica-Zeitschrift für feministische Politikwissenschaft 24 (2015), S. 9-24, hier S. 10.

94 Jinthana Haritaworn: „Am Anfang war Audre Lorde. Weißsein und Machtvermeidung in der queeren Ursprungsgeschichte* «, in: Femina Politica - Zeitschrift für feministische Politikwissenschaft (2005), S. 23-35, hier S. 28.

95 Ebd., S. 31.

96 B. Bargetz/G. Ludwig: Bausteine einer queerfeministischen politischen Theorie, S. 10.

97 Ebd., S. 9 . 
Geschlecht und der begrifflichen Einführung der »Heterosexuellen Matrix « auch die für die »Queer Studies« zentrale Theoretisierung des Verhältnisses von Geschlecht und Sexualität voran. Butler behauptet, dass nicht nur das soziale Geschlecht - gender - konstruiert sei, sondern auch das biologische Geschlecht - sex-diskursiv hergestellt werde. Mit dem Konzept der »Heterosexuellen Matrix« beschreibt sie ein hegemoniales MachtWissens-Regime oder auch Dispositiv, durch das vergeschlechtlichte Subjekte konstituiert werden. ${ }^{98} \mathrm{Neu}$ ist dabei nicht nur, dass das biologische Geschlecht als diskursiv hergestellt verstanden wird, sondern auch, dass Butler darauf verweist, wie in hegemonialen Geschlechter-Diskursen das biologische Geschlecht, die Geschlechtsidentität und das Begehren aufeinander bezogen sind und sich wechselseitig konstituieren: Dem biologischen Geschlecht folgt im hegemonialen Macht-Wissens-Regime der Heterosexuellen Matrix eindeutig die Geschlechtsidentität, die wiederum erfordert, dass das gegenteilige Geschlecht begehrt werden muss. ${ }^{99}$ Von dieser konstitutiven Verknüpfung von Geschlecht, Geschlechtsidentität und Begehren ausgehend, plädiert Butler auch für die Zusammenführung des feministischen und queeren Projekts. ${ }^{100}$

Diesen Überlegungen von Judith Butler, die mitunter als Bruch in der Theoretisierung von Geschlechterverhältnissen gelten, geht das Denken von lesbischen Theoretiker*innen, wie beispielsweise Monique Wittig und Adrienne Rich, voraus. So hat beispielsweise Monique Wittig bereits 1979 in ihrem Aufsatz »The Straight Mind « ${ }^{101}$ darauf verwiesen, dass Geschlecht und Begehren konstitutiv miteinander verknüpft sind. Die Lesbe - so Wittigs Argumentation - ist keine Frau, weil ihr Begehren nicht auf einen Mann ausgerichtet ist. ${ }^{102}$ Neben MoniqueWittig gelten auch die Überlegungen der lesbischen Theoretikerin und Dichterin Adrienne Rich, wie sie sie in dem 1980 erschienenen Artikel »Compulsory Heterosexuality and Lesbian Existence ${ }^{103}$ ausführt, als wegweisend für queere Theoriebildung. Rich arbeitet in diesem Essay die Zwänge der Institution der Heterosexualität heraus und führt das lesbische Kontinuum ein. Indem Heterosexualität begünstigt und privilegiert und Homosexualität demgegenüber sanktioniert wird, werden Frauen - so Richs These - in die Heterosexualität hineingezwungen. Das lesbische Kontinuum ist Richs Versuch, die Beziehungen zwischen Frauen auf all ihren Ebenen - nicht nur der sexuellen - fassbar zu machen. ${ }^{104}$

Volker Woltersdorff: »Queer Theory und Queer Politics«, in: Utopie kreativ 156 (2003), S. 914-923, hier S. $917 f$.

99 J. Butler: Körper von Cewicht, S. $326 f$.

100 Ebd., S. 329.

101 Monique Wittig: The Straight Mind and Other Essays, Boston: Beacon Press 1992.

102 B. Bargetz/G. Ludwig: Bausteine einer queerfeministischen politischen Theorie, S. 12. Es wird allerdings diskutiert, ob die Art, wie Wittig die Kategorie Lesbe einsetzt, identitätspolitisch ist oder ob es sich dabei um eine soziale Positionierung handelt. Wittig hebt in ihren Arbeiten auch die Verknüpfung von Sexualität, patriarchalen und kapitalistischen Verhältnissen hervor, indem sie aufzeigt, dass Heterosexualität die Reproduktion der Menschen durch Frauen grundlegend strukturiert.

103 Adrienne Rich: »Compulsory Heterosexuality and Lesbian Existence«, in: Women: Sex and Sexuality 5 (1980), S. 631-660.

104 M.-J. Barker/J. Scheele: Queer, S. 48f. 
Diese Überlegungen, gekoppelt mit der Kritik an der Repressionshypothese, wie sie Michel Foucault in seinem 1976 erschienenen Werk »Sexualität und Wahrheit 1« ausführt, führte Judith Butler weiter, um aufzuzeigen, wie Geschlechternormen unsere Körper regulieren. ${ }^{105}$ Die Macht, die die Menschen mittels wahrer Diskurse zur Sexualität reguliert - so Foucaults prominente Kritik an der Repressionshypothese - sei nicht einfach repressiv, sondern im Gegenteil insofern produktiv, als sie Subjekte konstituiert. ${ }^{106}$ Daran anschließend betont Judith Butler, insbesondere in den Arbeiten, die auf »Gender Trouble« folgten, dass die Regulierungen von gender nicht einfach auf ein ihnen vorgängiges Subjekt wirken, sondern dieses gerade in und durch die Regulierung selbst hergestellt wird. Entsprechend seien »die regulatorischen Diskurse, die das Subjekt von Gender formen, genau diejenigen [...], die das zur Debatte stehende Subjekt benötigen und es hervorrufen. ${ }^{107}$ Damit wird gender als Norm nicht als eine Macht verstanden, die über negative Einschränkungen reguliert, sondern als ein $\mathrm{Me}$ chanismus, der über eine Anzahl positiver Kontrollen funktioniert. ${ }^{108}$ Über die normative Komponente schreiben sich, Butlers Ausführungen zufolge, Machtverhältnisse in die Konstruktionen von Geschlecht und Begehren ein. An Butler, aber auch an andere Überlegungen anschließend, spielt auch für queere Theorie und Praxis die Auseinandersetzung mit Normativität eine wichtige Rolle. Anstatt von Zwangsheterosexualität zu sprechen, wie Rich es noch getan hat, schließt der für die queere Theorie und Praxis zentrale Begriff der Heteronormativität an ein komplexeres Machtverständnis an.

\section{Von der Heteronormativität zur Homonormativität}

Michael Warner benutzt in seiner Einleitung zum 1991 erschienenen Buch »Fear of a Queer Planet « als erster den Begriff »heteronormativity «. ${ }^{109}$ Bereits bei der ersten Verwendung des Begriffes knüpfte er diesen eng an das queere Projekt, indem er ebendieses zum Gegenspieler der Heteronormativität machte. Heteronormativität kann - so seine Aussage - nur durch die Imagination einer queeren Welt überwunden werden: »Even when coupled with a toleration of minority sexualities, heteronormativity can be overcome only by actively imagining a necessarily and desirably queer world. ${ }^{110}$ Normativitätskritik gilt entsprechend schon von Anfang an als ein zentraler Einsatzpunkt queerer Politiken, ${ }^{111}$ und auch queere Theorien beschäftigen sich grundlegend mit der

105 Auch psychoanalytische Impulse von Lacan oder Differenzfeminist"innen wie Irigaray haben Butlers Auseinandersetzungen geprägt.

106 Michel Foucault: Der Wille zum Wissen. Sexualität und Wahrheit 1, Frankfurt a.M.: Suhrkamp Verlag 2019 [1976], S. 93-102.

107 Judith Butler: Die Macht der Geschlechternormen und die Grenzen des Menschlichen, Frankfurt a.M.: Suhrkamp Verlag 2012, S. 72.

108 Ebd., S. 87.

109 Peter Wagenknecht:»Was ist Heteronormativität? Zu Geschichte und Cehalt des Begriffs«, in: Jutta Hartmann/Bettina Fritzsche/Christian Klesse et al. (Hg.), Heteronormativität. Empirische Studien zu Geschlecht, Sexualität und Macht, Wiesbaden: Springer Verlag 2007, S. 17-34, hier S. 18.

110 Michael Warner: Fear of a Queer Planet. Queer Politics and Social Theory, Minneapolis: University of Minnesota Press 1991, XVI.

111 V. Woltersdorff: Queer Theory und Queer Politics, S. 923. 
Frage, wie Subjekte jenseits der heterosexuellen Norm denkbar sind. ${ }^{112}$ Mit dem Begriff Heteronormativität wird ein unsere Gesellschaft durchziehendes Macht-WissensRegime beschrieben, das die zumeist unsichtbare Norm festschreibt, dass das gegenteilige Geschlecht zu begehren ist. Heteronormativität beschreibt - so Sabine Hark ein Ensemble von Verhältnissen, das ein »Gefühl der Richtigkeit« herstellt und Institutionen, Wissensordnungen aber auch Körper und Lebenswelten strukturiert. ${ }^{113}$

Auch der Staat wird in queeren Theorien häufig als heteronormativ strukturiert verstanden. Dementgegen gehe es »queer« gerade darum, Strategien zu entwickeln, um Heteronormativität jenseits von Forderungen nach rechtlicher Anerkennung unterlaufen zu können. ${ }^{114}$ Das queere Verhältnis zum Staat bleibt dabei allerdings paradox: Einerseits wird er als ein Ort verstanden, an dem (heteronormative) Ungerechtigkeiten hergestellt werden, andererseits wird ihm auch die Fähigkeit zugesprochen, $\mathrm{zu}$ schützen und zu unterstützen. ${ }^{115}$ Im Kampf um Rechte und Anerkennung laufen queere/homosexuelle Politiken aber auch Gefahr, selbst normativ zu sein. Dass Heteronormativität als alleinige Kategorie der Machtkritik zu kurz greift, zeigen Analysen, die auf queere Kompliz*innenschaft mit neoliberalen Strukturen aufmerksam machen. Um diese queere oder auch homosexuelle Normativität zu fassen, folgte dem Begriff der Heteronormativität der Begriff der Homonormativität. ${ }^{116}$ Allerdings beschreibt Homonormativität nicht eine analog zur Heteronormativität verstandene Norm der Homosexualität, die alle gesellschaftlichen Bereiche durchzieht, sondern queere Politiken der Anpassung an ein neoliberales Regime.

Die Bezeichnung Homonormativität geht auf den 2002 erschienenen Aufsatz »The New Homonormativity: The Sexual Politics of Neoliberalism« der US-amerikanischen Historikerin und Geschlechterforscherin Lisa Duggan zurück. ${ }^{117}$ Duggan beschreibt in diesem Aufsatz, wie sich in der Geschichte der Schwulen- und Lesbenbewegungen die Forderungen in Bezug auf das »Private« und »Öffentliche« verändert haben. Zwischen der »Homophilen Bewegung « der 1950er- und 60er-Jahre und der »Gay Liberation« in den 1970er-Jahren habe es zwar Konflikte gegeben, ob assimilatorische oder konfrontative politische Strategien gefahren werden sollten; in Bezug auf das Verhältnis von

112 Antke Engel: Bilder von Sexualität und Ökonomie. Queere kulturelle Politiken im Neoliberalismus, Bielefeld: transcript Verlag 2009, S. 18.

113 Sabine Hark: »Heteronormativität revisited. Komplexität und Grenzen einer Kategorie«, in: Barbara Paul/Lüder Tietz (Hg.), Queer as ... - Kritische Heteronormativitätsforschung aus interdisziplinärer Perspektive, Bielefeld: transcript Verlag 2016, hier S. $2 f$.

114 Lisa Duggan: »Queering the State«, in: Social Text (1994), S. 1-14, hier S. 3.

115 Heike Raab: »Aspekte queerer Staatskritik - Heteronormativität, institutionalisierte Identitätspolitiken und Staat«, in: Femina Politica - Zeitschrift für feministische Politikwissenschaft 14 (2005), hier S.60. Allerdings bleibt in queeren Forderungen nach Schutz, Anerkennung und Unterstützung der Staat der Ort, der entscheidet, welches Leben schützenswert ist und welches nicht. Vgl. Judith Butler: Haß spricht. Zur Politik des Performativen, Frankfurt a.M.: Suhrkamp Verlag 2016, S. 162.

116 Lisa Duggans Aufsatz spricht zunächst vor allem von spezifisch konservativen schwulen Politiken, die sich vermutlich nicht als »queer« bezeichnen würden.

117 Lisa Duggan: »The New Homonormativity«, in: Russ Castronovo/Dana D. Nelson/Donald E. Pease et al. (Hg.), Materializing Democracy: Toward a Revitalized Cultural Politics, North Carolina: Duke University Press 2002, S. 175-194. 
Sexualität und Öffentlichkeit beziehungsweise Privatheit seien die zwei Bewegungen aber eine ähnliche Linie gefahren. Es ging beiden um das Recht auf sexuelle Privatheit und eine Ausweitung des schwul-lesbischen öffentlichen und institutionalisierten Lebens. Einen Bruch mit diesen Politiken, den Duggan mit dem Begriff der Homonormativität beschreibt, verortet sie in den 1990er-Jahren. Anknüpfend an die konservativen »Anti-Gay«-Politiken der 8oer-Jahre, die versuchten, Homosexualität in das Private zu drängen, sowie ausgehend von Monogamie als eine Antwort, die mitunter auf die Aids-Krise ${ }^{118}$ gegeben wurde, sei in den 1990er-Jahren »a strain of gay moralism« entstanden. ${ }^{119}$ Diese neuen homosexuellen Politiken, wie Duggan sie bezeichnet, forderten eine Zone der häuslichen Privatheit ein, die mit konservativen Zielen und neoliberaler staatlicher Neutralität konform sind. Am Beispiel der »Gay Marriage«-Strategie des »Independent Gay Forums (IGF)« und der Aussagen eines der prominentesten Vertreter dieser Gruppe, Andrew Sullivan, führt Duggan aus, wie Homosexualität entpolitisiert und ins Private verschoben wird:

»But it says something about the unique status of homosexuals in our society that we now have to be political in order to be prepolitical. Our battle, after all, is not for political victory but for personal integrity. In the same way that many of us had to leave our families in order to join them again, so now as citizens, we have to embrace politics if only ultimately to free from it. ${ }^{120}$

Das, was Duggan unter »the new gay politics « zusammenfasst, ist eine Forderung nach öffentlicher Anerkennung einer entpolitisierten Privatheit. Diese »neue Homonormativität « beschreibt sie als antidemokratisch, entpolitisiert und neoliberal: "All of this adds up to a corporate culture managed by a minimal state, achieved by the neoliberal privatization of affective as well as economic and public life. Welcome to the New World Order! Coming soon to a mainstream near you. «21

Auch wenn ein großer Teil queerer Politiken nicht derart entpolitisiert und konservativ wie das von Duggan bemühte Beispiel des "IGF« agiert, wird auch diesen eine enge Verknüpfung mit neoliberalen Diskursen vorgeworfen. Antke Engel hebt beispielsweise hervor, dass gerade die individuellen Freiheits- und Selbstbestimmungsversprechen, aber auch die vielfältigen sexuellen und vergeschlechtlichten Identitätsangebote, die »queer hervorbringt, hervorragend mit neoliberalen Denkmustern harmonieren: "Ich möchte die These vertreten, dass neoliberale Diskurse eine Pluralisierung sexueller Subjektivitäten und Lebensformen forcieren, weil damit eine Ideologie der freien Gestaltbarkeit des eigenen Lebens, inklusive Körper und Selbst, versinnbildlicht werden kann. « ${ }^{122}$ Queere Politiken - so Engels Fazit - , die ausschließlich gegen Normativitäten agieren, können neoliberalen Denkmustern nicht entkommen. Die Politiken der Vervielfältigung, die das Ziel haben, Sichtbarkeit zu schaffen und »queer« offenzuhalten,

Eine Forderung nach einer monogamen Lebensführung grenzte sich von einer schwulen Promiskuität ab, der die Verbreitung von Aids mitunter zugeschrieben wurde.

121 Ebd., S. 190.

122 A. Engel: Bilder von Sexualität und Ökonomie, S. 26. 
laufen speziell Gefahr, neoliberal vereinnahmt zu werden. Die vielen dort produzierten neuen Identitätsformen würden bestens in neoliberale Konsum- und Selbstunternehmer*innen-Logiken passen. ${ }^{123}$ Doch mit dem Begriff der Homonormativität endet die Kritik an queeren Verstrickungen mit hegemonialen Verhältnissen nicht.

\section{Homonationalismus: Die neuen death-figures}

Eine weitere Denkbewegung, die an den Begriff der Homonormativität anschließt, den Fokus aber auf nationalistische Tendenzen legt, ist die von Jasbir Puar eingeführte Bezeichnung des »Homonationalismus«. Mit diesem Begriff will Puar die Spannung zwischen der zunehmenden Anerkennung und wirtschaftlichen Integration von Homosexualität im globalen Norden und den Subjekten, auf deren Kosten diese Integration geht, nämlich den »neuen Perversen« - »the muslim terrorists « -, beschreiben: »I argue that the contemporary U.S. heteronormative nation actually relies on and benefits from the proliferation of queerness, especially in regard to the sexually exceptional homonational and its evil counterpart, the queer terrorist of elsewhere. « ${ }^{124}$ Puar spricht im Zusammenhang mit Homonationalismus auch von einem Wandel des Verhältnisses von Nationalstaatlichkeit, Sexualität und Begehren: Waren es in den 1980er-Jahren die Homosexuellen, die als death-figures der Aids-Krise biopolitisch reguliert wurden, ist in homonationalistischen Zeiten, Puar zufolge, die alte Unterscheidung zwischen den "guten « heterosexuellen Staatsbürger*innen und den »schlechten « homosexuellen Staatsbürger*innen so nicht mehr gültig. Stattdessen werden ideale queere/homosexuelle Staatsbürger*innen und ein "gay-friendly« Staat in Abgrenzung zum »neuen Perversen $«$ - der muslimische Terrorist - konstruiert. ${ }^{125}$ Queere Politiken und Lebensweisen werden so, wie Puar am Beispiel von LGBTIQ-Menschenrechtsinterventionen im Iran aufzeigt, »zum Alibi« nationalstaatlicher und imperialistischer Politiken. In der Einleitung zu ihrem Buch »Terrorist Assemblage« fragt sie, warum der Westen ausgerechnet jetzt und genau an diesem Ort (Iran) anfange, queere Kämpfe zu unterstützen, wo doch auch schon früher und an anderen Orten Menschen aufgrund ihrer Homosexualität mit dem Leben bezahlt haben:

»Faisal Alam notes, that three Nigerian shomosexuak men were sentenced to be stoned to death earlier that summer elicited no such global indignation. Nor have these abuses elicited so much response from Igbtiq groups in the past. Along these lines, there were no protests in May 2004 when the circulation of photos of the torture practices at Abu Chraib exhumed the revolting homophobia of the U.S. military. As iglhrc's director Paula Ettelbrick asks, >Why now? Why just Iran?« ${ }^{126}$

Die Antwort ist für Puar eindeutig: Diese Politiken sind verknüpft mit geplanten USmilitärischen Aktionen. Indem die Homophobie in der sogenannten muslimischen Welt offengelegt und angeprangert wird, können Puar zufolge US-imperialistische Interventionen legitimiert werden. Das muslimische Subjekt wird zum neuen »Anderen«,

\footnotetext{
123 K. Linkerhand: Treffpunkt im Unendlichen, S. $56 f$.

124 J. K. Puar: Terrorist Assemblages, XXV.

125 Ebd., xxi.

126 Ebd., XI.
} 
durch das neo-koloniale Politiken gerechtfertigt werden. ${ }^{127}$ Dabei geht es Jasbir Puar nicht darum, zu leugnen, dass es Homophobie in muslimisch geprägten Ländern gibt, sondern darum, die Frage zu stellen, warum zu einem bestimmten Zeitpunkt über Homophobie - beispielsweise im Iran - gesprochen wird.

Nikita Dhawan resümiert, dass die Homonationalismus-Kritik von Puar und anderen dazu beigetragen habe, dass queere Politiken im globalen Norden den eigenen Rassismus und die Kompliz*innenschaft mit neoliberalen und neokolonialen Diskursen in den Blick nehmen. Doch sie verweist auch auf die Leerstellen einer Homonationalismus-Kritik, wie sie Puar eingeführt hat: Ihr einseitiges Verständnis von Macht und Gewaltausübung führt, Dhawan zufolge, zu einer »spiegelbildlichen Wiederholung « postkolonialer Verhältnisse. ${ }^{128}$ Puars Perspektive spreche queeren Menschen im globalen Süden jegliche Form von Handlungsmacht $a b$, indem sie sie einseitig als Opfer homonationalistischer Politiken darstelle. Zudem mache es der »ausschließliche Fokus auf westlichen Rassismus« unmöglich, »heterosexistische Gewalt, die >queers in und aus dem globalen Süden erfahren«, zu benennen. ${ }^{129}$ Ähnlich wie in Spivaks Ausführungen die Witwen zwischen den Ideologien der Briten, die sie zu retten vorgeben, und denen der »einheimischen « Männer, die ihnen die Möglichkeit geben wollen, eine gute Ehefrau zu sein, zerrieben werden, so werden auch Queers aus dem globalen Süden zerrieben: Sie stehen zwischen den homophoben und misogynen Tendenzen ihrer eigenen Gemeinschaften und der Dominanzgesellschaft und den rassistischen Praxen im Westen ${ }^{130}{ }^{130}$ Dementgegen fordert Dhawan eine multidirektionale Kritik, die beide Seiten der Medaille beleuchtet. ${ }^{131}$ Mit Bezug auf eine mögliche Antwort auf Gewalt gegen Migrant*innen in Europa - die ein ähnliches Dilemma hervorbringt - resümieren Dhawan und Castro Varela: »Eine gute Lösung scheint es nicht zu geben und dennoch muss beständig beides gemacht werden: das Offenlegen rassistischer Praxen und die Thematisierung von Gewalt gegen Frauen (Queers). «32

Dhawan vertritt aber auch die These, dass die queeren und linken Politiken im globalen Norden zugrunde liegende Staatsphobie $\mathrm{zu}$ einer einseitigen Homonationalismus-Kritik, wie sie Puar vertritt, beitrage. Puars einseitiger Blick auf den Staat als Ort, der die »Perversen« - seien es die Homosexuellen oder queeren Terrorist*innen - reguliert, klammert aus - so Dhawans Vorwurf -, dass der Staat »Gift und Medizin« zugleich ist. Puar ignoriere, was Michel Foucault als linke Staatsphobie bezeichnet. ${ }^{133}$ Diese Staatsphobie auch auf postkoloniale Kontexte anzuwenden, sei gefährlich, weil Queers im globalen Süden oftmals nicht in gleicher Weise durch die staatliche Rechtsprechung geschützt sind: "Damit werden Absicherungen

127 Ebd., S. 4.

128 Nikita Dhawan: »Homonationalismus und Staatsphobie: Queering Dekolonisierungspolitiken, Queer-Politiken dekolonisieren«, in: Femina Politica - Zeitschrift für feministische Politikwissenschaft 24 (2015), S. 38-51, hier S. 42.

130 Ebd.

131 Ebd., S. 39.

132 M. d. M. Castro Varela/N. Dhawan: Die Migrantin retten!?, S. $25 f$.

133 Diese hat sich Foucault zufolge in Abgrenzung zu den Erfahrungen mit einem faschistischen und totalisierenden Staat im globalen Norden etabliert. 
verweigert, die postkoloniale Queers auf der privilegierten Seite der Transnationalität genießen, da sie an Orten leben, an denen Homosexualität entkriminalisiert wurde. « ${ }^{134}$ In postkolonialen Kontexten gilt es stattdessen, Dhawan zufolge, vielmehr in einer gramscianisch-spivakischen Perspektive »subalternen Gruppen Zugang zum Staat zu ermöglichen «. ${ }^{135}$

Die queer-theoretische Bewegung von der Heteronormativität zum Homonationalismus zeigt auf, wie sich die Aufgabe, vor der queere Politiken stehen, verkompliziert hat. Ging es zunächst ausgehend von dem Begriff der Heteronormativität darum, Normen, die Queers zu vergeschlechtlichten Subjekten machen und regulieren, zum Ort des Politischen zu machen, hat der Begriff der Homonormativität gezeigt, dass es aufgrund neoliberaler Verstrickungen nicht ausreicht, einfach nur die heterosexuelle Norm anzuprangern. Neoliberale Regierungstechniken werden immer feingliedriger und die diskursiven Verwicklungen mit queeren Politiken entsprechend engmaschiger. ${ }^{136}$ Die neue Normalität beteiligt sich mitunter zudem an nationalistischen Strategien des Othering und geht so auf Kosten der neuen death-figures. Trotz zunehmender Anerkennung sind Queers allerdings auch im globalen Norden immer noch diejenigen, die im Unterschied $\mathrm{zu}$ denen, die tolerieren, toleriert werden. ${ }^{137}$ 
